\title{
IL VOTO DI APPROVAZIONE: \\ IL DOPPIO TURNO IN UN TURNO SOLO \\ di Roberto D'Alimonte
}

\section{Condorcet e il sistema elettorale ottimale}

Esiste un sistema elettorale ottimale? La risposta convenzionale è negativa. Ogni sistema elettorale ha pregi e difetti. La scelta del sistema dipende da numerosi fattori, e in primo luogo dagli obiettivi che si vogliono conseguire. Non esiste quindi un sistema intrinsecamente superiore agli altri. Questa risposta vale se la scelta è tra sistemi proporzionali e sistemi maggioritari/ uninominali. Non vale però se dobbiamo scegliere all'interno della categoria dei sistemi maggioritari/uninominali.

In questo caso esistono dei criteri per valutare se un dato sistema elettorale è superiore ad altri ${ }^{1}$. Il più importante - a me pare - è questo: dato un numero di candidati maggiore di due, un sistema elettorale maggioritario/uninominale è migliore di altri se rende più probabile la scelta del candidato preferito dalla maggioranza degli elettori. Posto così, però, il criterio è ancora troppo generico perché non specifica cosa si debba intendere con l'espressione «maggioranza degli elettori». Come si può determinare qual è la volontà della maggioranza degli elettori quando i candidati sono più di due e nessuno ottiene il $50 \%$ dei voti più uno?

Con un sistema elettorale maggioritario a un turno il problema si risolve con l'elezione del candidato che ottiene più voti (criterio della maggioranza relativa). Ma questo criterio ha un limite rilevante. Prendiamo tre candidati (A, B, C) e supponiamo che vinca $A$. Se tra i perdenti ci fosse un candidato (B) che messo a confronto con $A$ in un ballottaggio (quindi senza la partecipazione del terzo concorrente) ottenesse più voti di $\mathrm{A}$,

\footnotetext{
1 Per un'analisi formale di questi criteri vedi Nurmi (1983).
}

RIVISTA ITALIANA DI SCIENZA POLITICA / a. XXIV, n. 2, agosto 1994 
sarebbe difficile sostenere che A è la scelta preferita dalla maggioranza degli elettori. In realtà, la maggioranza preferisce $B$ ad A. Se poi B fosse messo a confronto anche con $C$ (senza la partecipazione al voto di $\mathrm{A}$ ) e ottenesse più voti di $\mathrm{C}$, allora il responso dell'elettorato sarebbe assolutamente chiaro: tra $\mathrm{A}, \mathrm{B}$ e C, la maggioranza preferisce $B$. Eppure, con il sistema maggioritario ad un turno il vincitore è $A$.

Il criterio «ideale» quindi per scegliere un candidato in un contesto di elezioni con più di due concorrenti è questo: il candidato preferito dalla maggioranza degli elettori è quello che, in una serie di votazioni binarie (ballottaggi) con ciascuno degli altri concorrenti, ottiene la maggioranza dei voti. $\mathrm{Ci}$ riferiremo al candidato che soddisfa questo criterio come al «candidato Condorcet»o «candidato di maggioranza» ${ }^{2}$.

Definito il criterio restano due problemi:

1) esiste sempre questo candidato?

2) se esiste, quale sistema elettorale assicura meglio che egli venga eletto?

La seconda domanda può essere riformulata anche in questi termini: posto che non sia possibile far votare gli elettori sulla base di una serie di scontri binari, quale sistema si avvicina di più al criterio di Condorcet? ${ }^{3}$

Questo saggio è dedicato soprattutto alla seconda di queste domande. Ma una risposta alla prima va comunque data. E la risposta è negativa. Non sempre esiste un candidato di maggioranza nella definizione che abbiamo dato qui a questo concetto, vale a dire un candidato che soddisfa il criterio di Condorcet. È questo uno dei significati del famoso «teorema della impossibi-

2 Il criterio si deve al matematico francese Condorcet (1785) che lo contrapponeva al metodo di Borda (1781). Per una ricostruzione storica della genesi di questo criterio vedi Black (1958). Nella letteratura sulle procedure di voto si parla di due criteri di Condorcet. Infatti, al criterio citato - che è quello più noto - se ne aggiunge un altro che è l'esatto contrario. Mentre il primo definisce il candidato «Condorcet-vincente», il secondo definisce il candidato «Condorcet-perdente». Quest'ultimo è il candidato che «in un contesto di elezioni con più di due concorrenti è sconfitto in una serie di votazioni binarie da tutti gli altri candidati». Nel corso di questo articolo faremo riferimento solo al primo di questi criteri e parleremo di «candidato Condorcet» invece che di «candidato Condorcet-vincente» 0 «vincitore-Condorcet».

3 In realtà, non è necessario ricorrere ad una serie di votazioni binarie per individuare il candidato Condorcet. Assumendo l'ipotesi della sincerità del voto, è sufficiente disporre delle scale di preferenza degli elettori relativamente a tutti i candidati in competizione. Da questo punto di vista il sistema elettorale reale che più si avvicina al «metodo Condorcet» è il voto alternativo, vedi nota 7 . 
lità generale» di Arrow (1963). È possibile infatti che, dati tre o più candidati, la regola di maggioranza produca il cosiddetto "paradosso del voto», vale a dire una serie di maggioranze cicliche (un circolo vizioso) invece di una soluzione di equilibrio stabile con un unico vincitore ${ }^{4}$.

$\mathrm{Ma}$ se un candidato di maggioranza esiste, quale sistema elettorale concreto dobbiamo utilizzare per identificarlo? A questa domanda si può rispondere solo confrontando vantaggi e svantaggi dei diversi sistemi elettorali maggioritari/uninominali. In questa sede prenderemo in considerazione (senza pretendere di fare una comparazione sistematica) alcuni di questi sistemi: il maggioritario a un turno (che chiameremo amche maggioritario semplice), il doppio turno, il voto di approvazione. I primi due sono noti. Il terzo è una «invenzione» degli ultimi quindici anni. È soprattutto all'analisi del voto di approvazione che questo articolo è dedicato. Ma, prima di entrare in argomento, facciamo una digressione utilizzando un esempio concreto tratto dalle elezioni politiche del 27-28 marzo 1994 per spiegare meglio i concetti introdotti qui.

Berlusconi, Spaventa e Michelini: chi era il «candidato Condorcet» nel collegio di Roma-Centro?

Prendiamo il collegio di Roma-Centro. I candidati erano quattro di cui solo tre competitivi sulla carta. I risultati sono quelli della tab. 1.

Berlusconi ha vinto, ma era Berlusconi il candidato preferito dalla maggioranza degli elettori del collegio, vale a dire il «candidato Condorcet»? In uno scontro diretto con Spaventa (senza Michelini) e con Michelini (senza Spaventa) sarebbe riuscito ad ottenere la maggioranza assoluta dei voti validi?

Per rispondere dovremmo avere una informazione più completa sulla struttura di preferenze degli elettori di Spaventa e di Michelini. In particolare, dovremmo conoscere le loro seconde preferenze. Il sistema elettorale adottato non lo consente. Non possiamo quindi sapere con certezza quanti elettori di Michelini avrebbero preferito Spaventa a Berlusconi. Soprattutto non

${ }^{4}$ Il paradosso si verifica quando $A$ batte $B, B$ batte $C$, ma $C$ batte $A$. In questo caso non esiste un «vincitore Condorcet». Anzi, non esiste nessun vincitore. 
TAB. 1. Risultati elettorali del collegio di Roma-Centro (Camera)

\begin{tabular}{|c|c|c|c|c|}
\hline Candidati & Contrassegni candidato & Voti validi & $\%$ Voti & Liste collegate \\
\hline Silvio Berlusconi & $\begin{array}{l}\text { Forza Italia, Centro Crist. Dem., } \\
\text { Polo Liberal Dem., Unione di } \\
\text { Centro, Alleanza Nazionale }\end{array}$ & 34.534 & 46,3 & $\begin{array}{l}\text { Forza Italia, Alleanza } \\
\text { Nazionale }\end{array}$ \\
\hline Luigi Spaventa & Progressisti & 29.914 & 40,1 & $\begin{array}{l}\text { PDS, Rifondazione Comunista, } \\
\text { PSI, Federazione dei Verdi } \\
\text { Alleanza Democratica, La Rete }\end{array}$ \\
\hline Alberto Michelini & Patto per l'Italia & 9.566 & 12,8 & Patto Segni \\
\hline Mirella Cece & $\begin{array}{l}\text { Movimento Europeo Liberal- } \\
\text { Cristiano «Giustizia e Libertà» }\end{array}$ & 593 & 0,8 & $\begin{array}{l}\text { Movimento Europeo Liberal- } \\
\text { Cristiano «Giustizia e Libertà» }\end{array}$ \\
\hline Totale & & 74.607 & 100,0 & \\
\hline
\end{tabular}

Fonte: Elaborazione dell'autore. 
sappiamo quanti elettori di Spaventa avrebbero votato a favore di Michelini in un ballottaggio tra lui e Berlusconi. Possiamo però fare delle ipotesi e ragionare su quelle. A fini esemplificativi, immaginiamo che la struttura di preferenze degli elettori del collegio fosse questa:

$\begin{array}{lll}\text { Spaventa } & \text { Michelini } & \text { Berlusconi } \\ \text { Michelini } & \text { Spaventa } & \text { Michelini } \\ \text { Berlusconi } & \text { Berlusconi } & \text { Spaventa }\end{array}$

Le percentuali di elettori con questi ordini di preferenza sono quelli della tabella 1 , cioè quelli reali. Rispetto alla tabella, abbiamo solo aggiunto delle ipotesi sulle seconde e terze preferenze degli elettori.

Con queste informazioni possiamo ora rispondere alla domanda su chi avrebbe vinto tra questi candidati nel caso in cui avessimo potuto ricorrere ad una serie di votazioni binarie. E la risposta è Michelini. Infatti, Michelini vincerebbe contro Spaventa potendo contare sui suoi voti (il $12,8 \%$ ) e su quelli dei sostenitori di Berlusconi che lo preferiscono a Spaventa; e vincerebbe anche contro Berlusconi essendo preferito a quest'ultimo dagli elettori di Spaventa. A questo punto, l'esito del confronto tra Spaventa e Berlusconi è irrilevante.

Qualcuno però potrebbe obiettare che, mentre è plausibile l'ipotesi che le seconde preferenze degli elettori di Spaventa e di Berlusconi sia Michelini, non è plausibile che la seconda preferenza degli elettori di Michelini sia Spaventa. Occorre chiedersi allora se il risultato degli scontri binari sarebbe cambiato se la seconda preferenza degli elettori di Michelini fosse stata Berlusconi, anziché Spaventa. La risposta è negativa. Il risultato sarebbe stato identico: la vittoria di Michelini. In questo caso, la seconda preferenza degli elettori di Michelini è irrilevante. Il «candidato Condorcet» resta Michelini. Infatti, il risultato non dipende dalla seconda preferenza degli elettori di Michelini, ma dalla seconda preferenza degli elettori di Spaventa e di Berlusconi, oltre che dalla loro propensione a votare Michelini ovvero ad astenersi in assenza del loro candidato favorito.

Nel caso concreto, è molto probabile che comunque Berlusconi fosse il «candidato Condorcet» per due ragioni. Primo, è molto probabile che un certo numero di elettori di Spaventa si sarebbe astenuto piuttosto che votare Michelini. Secondo, è possibile che alcuni elettori di Spaventa avrebbero preferito il 
laico Berlusconi al cattolico Michelini. Per l'una o l'altra di queste ragioni (o per entrambe), Berlusconi avrebbe vinto anche in una serie di ballottaggi. Se però rigettiamo sia l'ipotesi dell'astensionismo di sinistra che quella di uno spazio politico non lineare (con una dimensione religiosa che interseca l'asse sinistra-destra), allora la conclusione, solo apparentemente paradossale, è che il candidato meno votato - Michelini - era in realtà il candidato preferito dalla maggioranza degli elettori.

Certo, di fronte ad una conclusione simile possono sorgere dei dubbi sulla bontà del criterio di Condorcet. Ma la risposta a questi dubbi è la seguente: se, data una certa struttura di preferenze, un candidato Condorcet esiste, questo è il candidato complessivamente preferito, quello che rappresenta meglio l'insieme delle preferenze degli elettori. Un candidato diverso dal candidato Condorcet potrebbe essere il candidato preferito dalla minoranza più forte, ma non dalla maggioranza. Con un sistema maggioritario ad un turno, nel caso in cui il candidato vincente non ottenga la maggioranza dei voti, possiamo presumere che egli sia il candidato della maggioranza, ma sempre di presunzione si tratta. Una presunzione che diventa tanto più labile quanto più ci si allontana dalla soglia di maggioranza. Al «criterio della minoranza più forte» si potrà ricorrere quando non esiste un candidato di maggioranza, ma non prima ${ }^{5}$.

Che il problema non sia di poco conto è dimostrato dai risultati complessivi (parte uninominale) delle ultime elezioni politiche. Le tabb. 2 e 3 li riassumono in base alla percentuale dei voti (divisa per classi) ottenuti dai candidati vincenti nei collegi uninominali sia alla Camera che al Senato.

Solo il $32.5 \%$ dei deputati e il $6.9 \%$ (!) dei senatori eletti con il sistema maggioritario hanno ottenuto la maggioranza as-

5 È interessante ipotizzare cosa sarebbe successo a Roma-Centro con il sistema maggioritario a due turni. In caso di ballottaggio Michelini non sarebbe nemmeno passato al secondo turno, visti i risultati effettivi. La contesa sarebbe stata tra Spaventa e Berlusconi. Chi avrebbe vinto? Questa volta, a differenza del caso di votazioni binarie, la risposta dipende dalle seconde preferenze degli elettori di Michelini. Se Spaventa fosse stata la loro seconda preferenza (almeno per il $10 \%$ di loro), Spaventa avrebbe vinto. Altrimenti, il risultato sarebbe stato favorevole a Berlusconi. Ma il punto importante è un altro: col doppio turno, e non con il turno singolo, entrano in gioco le seconde preferenze degli elettori; in particolare quelle degli elettori i cui candidati favoriti sono poco competitivi o addirittura esclusi dal ballottaggio. Anche in questo caso, quindi, come nel caso del metodo di Condorcet, sono maggiori le probabilità di scegliere il candidato effettivamente preferito dalla maggioranza degli elettori perché sono maggiori le informazioni utilizzate dal sistema elettorale per arrivare alla scelta finale. 
ТАВ. 2. Eletti nei collegi uninominali della Camera per aggregazione politica e percentuale di voto

\begin{tabular}{|c|c|c|c|c|c|c|c|c|c|c|}
\hline \multirow[t]{2}{*}{ Aggregazioni } & \multicolumn{10}{|c|}{ Classi di voto } \\
\hline & $>60 \%$ & $60-55$ & $55-50$ & $50-45$ & $45-40$ & $40-35$ & $35-30$ & $<=30$ & Totali & $\%$ \\
\hline Forza Italia/Lega Nord & 17 & 25 & 43 & 38 & 23 & 15 & 2 & 1 & 164 & 34,5 \\
\hline Forza Italia/Alleanza Nazionale & 4 & 4 & 20 & 38 & 24 & 27 & 10 & 3 & 130 & 27,4 \\
\hline Alleanza Nazionale & & & & & & & 3 & 5 & 8 & 1,7 \\
\hline Patto Segni/Partito Popolare & & & & & & 1 & 1 & 2 & 4 & 0,8 \\
\hline Progressisti & 4 & 14 & 19 & 35 & 31 & 30 & 21 & 10 & 164 & 34,5 \\
\hline Altri & 3 & & 1 & & & & 1 & & 5 & 1,1 \\
\hline Totali & 28 & 43 & 83 & 111 & 78 & 73 & 38 & 21 & 475 & \\
\hline$\%$ & 5,9 & 9,1 & 17,5 & 23,4 & 16,4 & 15,4 & 8,0 & 4,4 & 100,0 & \\
\hline
\end{tabular}

Fonte: Elaborazioni dell'autore su dati del Ministero dell'Interno. 
TAB. 3. Eletti nei collegi uninominali del Senato per aggregazione politica e percentuali di voto

\begin{tabular}{|c|c|c|c|c|c|c|c|c|c|c|}
\hline \multirow[t]{2}{*}{ Aggregazioni } & \multicolumn{10}{|c|}{ Classi di voto } \\
\hline & $>60 \%$ & $60-55$ & 55.50 & $50-45$ & $45-40$ & $40-35$ & $35-30$ & $<=30$ & Totali & $\%$ \\
\hline Forza Italia/Lega Nord & & & 1 & 6 & 28 & 24 & 12 & 3 & 74 & 31,9 \\
\hline Forza Italia/Alleanza Nazionale & & & 1 & 1 & 8 & 23 & 13 & 8 & 54 & 23,3 \\
\hline Patto Segni/Partito Popolare & & & & & 1 & & 2 & & .3 & 1,3 \\
\hline Progressisti & & 1 & 11 & 14 & 15 & 21 & 30 & 5 & 97 & 41,8 \\
\hline Altri & 2 & & & & & 1 & 1 & & 4 & 1,7 \\
\hline Totali & 2 & 1 & 13 & 21 & 52 & 69 & 58 & 16 & 232 & \\
\hline$\%$ & 0,9 & 0,4 & 5,6 & 9,1 & 22,4 & 29,7 & 25,0 & 6,9 & 100,0 & \\
\hline
\end{tabular}


soluta dei voti validi. Al Senato il $54.7 \%$ è stato eletto con meno del $40 \%$ e il $31.9 \%$ con meno del $35 \%$. Alla Camera, le cose sono andate meglio, ma resta sempre il fatto che il $27.8 \%$ dei deputati è pur sempre stato eletto con meno del $40 \%$ dei voti. Quanti di questi candidati minoritari sono «candidati Condorcet»? La risposta, a mio avviso, non può che essere dubitativa. Sono troppi, soprattutto al Senato, i vincitori ben lontani dalla soglia del $50 \%$. In un contesto di elezioni con più di due candidati, questo è un limite grave del sistema maggioritario semplice. Col tempo questo limite può diventare meno stringente perché è probabile che il processo di apprendimento delle regole da parte degli elettori e dei partiti porti ad una semplificazione della struttura della competizione con l'eliminazione progressiva delle candidature non competitive. Ma non è detto; soprattutto in una situazione politica di persistente e tendenziale frammentazione politica, in cui il sistema maggioritario ad un turno potrebbe fornire un incentivo alla presentazione di candidature di bandiera e/o di ricatto anche solo allo scopo di dividere il voto di candidati competitivi.

In ogni caso, perché affidare alla variabile tempo un esito che può essere ottenuto con un diverso sistema elettorale? Prendiamo, per esempio, il doppio turno con ballottaggio. Non c'è alcun dubbio che con questo sistema molti candidati eletti il 27-28 marzo (in particolare, candidati progressisti) non avrebbero ottenuto il seggio perché non erano i candidati preferiti dalla maggioranza. Questa ipotesi non si basa solo sulla valutazione dell'influenza del sistema elettorale. Si basa anche sulla scelta dei partiti (di centro e di destra) di presentare ciascuno il proprio candidato e in qualche caso anche più di uno ${ }^{6}$. Ciò, però, non toglie forza all'argomento che il doppio turno avrebbe prodotto un risultato diverso ora consentendo la selezione di candidati «migliori», eletti grazie non solo alle prime preferenze degli elettori, ma anche alle seconde. Questo è il punto cruciale, il vantaggio più importante del doppio turno sul turno singolo:

6 Alla Camera, nelle circoscrizioni delle Marche, Abruzzi e Campania 2, Forza Italia e Alleanza Nazionale hanno presentato ciascuno il proprio candidato. La conseguente divisione del voto di destra ha consentito la vittoria di molti candidati progressisti con percentuali di voto basse. Complessivamente, 31 dei 164 candidati progressisti eletti alla Camera e 35 dei 97 eletti al Senato sono stati eletti con percentuali di voto inferiori o uguali al $35 \%$. Il fenomeno si presenta anche nel caso del Polo delle Libertà e del Polo del Buon Governo, ma è meno pronunciato (vedi tabelle 2 e 3 ). 
esso sfrutta un numero di informazioni (le preferenze degli elettori) maggiore rispetto al turno singolo e quindi produce un risultato migliore perché il candidato vincente riflette in maniera più completa la struttura delle preferenze del corpo elettorale. $\mathrm{Da}$ questa caratteristica del doppio turno derivano analiticamente ed empiricamente altri vantaggi rilevanti. Tra i quali merita citare gli incentivi che esso offre alla presentazione di candidati moderati capaci di raccogliere un consenso il più ampio possibile e quindi una tendenza centripeta della competizione elettorale.

Il maggioritario a due turni, però, non è il solo sistema elettorale che utilizza le seconde preferenze degli elettori per definire la scelta del candidato preferito dalla maggioranza. Intanto, ci sono i sistemi «ad ordinamento delle preferenze», tra i quali il più noto è il voto alternativo ${ }^{7}$. Ma questi sono sistemi complessi. Un'alternativa relativamente semplice e poco nota è invece il voto di approvazione ${ }^{8}$.

\section{Il voto di approvazione: un'alternativa al doppio turno?}

L'idea di fondo è molto semplice. Dati $x$ collegi uninominali e $y$ candidati in ciascun collegio $(y>2)$, l'elettore può votare tutti $\mathrm{i}$ candidati che vuole. Il voto dato a ciascun candidato ha lo stesso peso. In altre parole, all'elettore non si chiede di ordi-

7 Con questo sistema si richiede all'elettore di disporre tutti $\mathrm{i}$ candidati in ordine di preferenza. Il candidato che ottiene il minor numero di «prime preferenze» viene eliminato e $\mathrm{i}$ suoi voti vengono trasferiti agli altri candidati che restano in corso e $\mathrm{i}$ cui nomi compaiono al secondo posto nelle scale di preferenza degli elettori il cui candidato maggiormente preferito (prima preferenza) è stato eliminato. Il processo di eliminazione e di ridistribuzione continua fino a quando un candidato ottiene la maggioranza assoluta dei voti validi. L'Australia è il paese in cui questo sistema elettorale è utilizzato da più tempo, in particolare per le elezioni della Camera dei Rappresentanti a livello federale, ma anche a livello di numerosi parlamenti sub-nazionali. Sull'esperienza australiana vedi Wright (1986).

8 L'idea è cominciata a circolare negli Stati Uniti nella seconda metà degli anni settanta. La trattazione più rigorosa e più sistematica si deve a Brams e Fishburn (1983), a cui si rinvia per una rassegna dettagliata della bibliografia sull'argomento. Questi due autori sono anche tra $\mathrm{i}$ più fervidi sostenitori della superiorità di questo sistema di votazione, che anzi definiscono come «la più significativa riforma elettorale del ventesimo secolo» $(1983,12)$. A quanto mi risulta, questo sistema non ha ancora trovato alcuna applicazione pratica. Ha suscitato però notevole interesse in circoli accademici dove ha trovato consensi, ma anche critiche. Per un'analisi critica, ma non tecnica, vedi Arrington e Brenner (1984) e la risposta di Brams e Fishburn (1984). Per un'analisi critica, ma più formale, vedi Niemi (1984). 
nare $\mathrm{i}$ candidati dal più preferito $\mathrm{al} \mathrm{meno} \mathrm{preferito,} \mathrm{come} \mathrm{nei} \mathrm{si-}$ stemi elettorali ad ordinamento delle preferenze. Gli si offre invece la possibilità di esprimere una o più preferenze tra quei candidati in competizione che egli considera in qualche misura accettabili e che quindi si sente di «approvare». Il candidato che ha avuto più voti ottiene il seggio. In questo modo, il vincitore è il candidato «approvato» dal maggior numero di elettori nel collegio. A nessun candidato può andare più di un voto. L'elettore può votare tutti i candidati che vuole (che approva), ma non può concentrare i suoi voti sul candidato che preferisce in assoluto.

Torniamo al collegio di Roma-Centro e simuliamo l'uso del voto di approvazione. Con tre candidati in corsa (continuiamo a non tener conto della quarta candidata), gli elettori possono esprimere un massimo di due preferenze. Va da sé che non possono approvare tutti $\mathrm{i}$ candidati perché in tal modo è come se non votassero per alcuno in quanto le tre preferenze si annullerebbero. Possono però votare per un solo candidato. Se tutti facessero così, il voto di approvazione equivarrebbe al sistema maggioritario ad un turno e il risultato sarebbe identico: il seggio a Berlusconi.

Assumiamo invece che tutti gli elettori di Spaventa decidano di avvalersi della opportunità offerta dal sistema e quindi esprimano una seconda preferenza e che nessuno degli elettori di Michelini e di Berlusconi faccia altrettanto. Se supponiamo che sia Michelini il candidato più accettabile tra i due e quindi il destinatario del secondo voto, Michelini sarebbe il vincitore. Per i sostenitori di Berlusconi la situazione decisionale si presenta allo stesso modo: se votano solo Berlusconi, potrebbe vincere Spaventa. Meglio allora approvare anche Michelini. In ogni caso quindi il risultato è la vittoria di Michelini, come nell'ipotesi di applicazione del metodo Condorcet. Michelini sarebbe stato eletto come il candidato complessivamente più gradito (o meno sgradito) alla maggioranza degli elettori.

Esiste però una differenza tra metodo Condorcet e voto di approvazione: con il primo non contano le seconde preferenze degli elettori di Michelini, come già abbiamo visto. Qualunque sia il loro secondo candidato, Michelini avrebbe prevalso. Con il voto di approvazione, la loro seconda preferenza è determinante, a meno di non assumere che essi siano indifferenti tra Spaventa e Berlusconi e quindi non approvino né l'uno né l'altro. Scartata però l'ipotesi dell'indifferenza, per chi avrebbero 
espresso il loro secondo voto? La domanda è esattamente la stessa che ci porremo con un sistema di doppio turno: per chi avrebbero votato al secondo turno gli elettori di Michelini se il loro candidato non fosse stato presente? Da qui si vede chiaramente che il secondo voto (di approvazione) è l'equivalente funzionale del secondo turno e produrrebbe gli stessi effetti. Nell'uno e nell'altro caso (e contrariamente al sistema maggioritario semplice) sono le seconde preferenze degli elettori a fare la differenza. Ma analizziamo ora in maniera più sistematica $\mathrm{i}$ meriti del voto di approvazione?.

Date certe condizioni (che vedremo in seguito), il voto di approvazione garantisce l'elezione del candidato Condorcet. In precedenza abbiamo fatto notare che con il sistema maggioritario ad un turno è del tutto possibile che venga eletto il candidato preferito da una minoranza forte anche se in realtà è il candidato meno preferito dalla maggioranza degli elettori del collegio. Questo risultato paradossale si verifica soprattutto quando due $o$ più candidati si dividono il voto del centro permettendo ad un candidato estremo di vincere con una quota di voti relativamente bass $a^{10}$. Nelle ultime elezioni questo caso si è verificato in numerosi collegi, come suggeriscono i dati delle tabelle 2 e 3 . Con il voto di approvazione questo non sarebbe accaduto perché gli elettori dei candidati di centro avrebbero comunque potuto esprimere anche un secondo voto per un altro dei candidati moderati in corsa, alzando la soglia dei voti necessari per ottenere il seggio e impedendo così la vittoria del candidato «eccentrico».

Il voto di approvazione offre a partiti e candidati un incentivo a offrire immagini e programmi capaci di attirare un sostegno il

9 Seguiamo qui (con alcune varianti) l'elenco che ne fanno Brams e Fishburn (1983) lasciando all'ultima parte di questo saggio l'analisi critica.

10 Utilizzando un modello della competizione elettorale in uno spazio unidimensionale, Cox (1985) ha dimostrato che «col voto di approvazione una posizione di equilibrio esiste in elezioni con molti candidati quando tutti $i$ candidati adottano la posizione dell'individuo mediano... e che questo equilibrio è unico quando $i$ candidati sono tre... Invece, con il sistema maggioritario a un turno non esistono posizioni di equilibrio quando i candidati sono più di due oppure le posizioni di equilibrio non sono di centro». Questo risultato rafforza l'argomento che il voto di approvazione tende a favorire la vittoria di candidati moderati (di centro) almeno nel caso in cui lo spazio politico è lineare $\mathrm{e} i$ candidati non sono più di tre. 
più vasto possibile invece di cercare l'appoggio di segmenti dell'elettorato con interessi forti e specifici, ma minoritari. Candidati radicali e/o minoritari difficilmente possono contare sul secondo voto dei sostenitori degli altri candidati. Ne consegue che in un confronto tra minoranze organizzate vince quella $i$ cui candidati sono più accettabili ai sostenitori delle altre. In teoria, anche il sistema maggioritario semplice dovrebbe favorire la presentazione di candidature con un vasto sostegno, ma spesso questo incentivo viene meno in un contesto di proliferazione delle candidature e di divisione del voto di centro. In questo caso non vince la minoranza più accettabile, ma quella più forte.

Il voto di approvazione favorisce l'espressione di un voto sincero. Con questo sistema gli elettori possono votare i candidati che preferiscono con la certezza che questa strategia è la loro strategia ottimale. In altre parole, non hanno alcun incentivo a votare candidati meno preferiti per evitare il successo di un candidato assolutamente sgradito $^{11}$. Questo non è vero con il sistema maggioritario a un turno. Prendiamo di nuovo il caso del collegio di Roma-Centro. I sostenitori di Michelini che avessero temuto fortemente una possibile vittoria di Spaventa avrebbero dovuto votare Berlusconi (il candidato più competitivo e meno sgradito), invece che votare il loro candidato preferito ${ }^{12}$. Questo è un esempio di come il sistema a un turno costringa gli elettori a comportarsi strategicamente (e quindi a fare calcoli di convenienza) per massimizzare l'utilità del loro voto. Col voto di approvazione i sostenitori di Michelini avrebbero potuto comunque votare il loro candidato e in aggiunta esprimere un secondo voto di approvazione per Berlusconi (cioè votare sinceramente) avendo la certezza che in questo modo il loro sostegno a $\mathrm{Mi}$ chelini non avrebbe favorito la vittoria di Spaventa dividendo il voto moderato.

Il voto di approvazione riduce il fenomeno del «voto sprecato» e conseguentemente tende a favorire la partecipazione elettorale.

"Un sistema elettorale che non gode di questa proprietà è il nuovo sistema elettorale per Camera e Senato. Sul punto vedi D'Alimonte e Chiaramonte (1993, 534-539).

${ }_{12}$ Una spia di questo fenomeno è il fatto che Patto Segni e Partito Popolare hanno ottenuto nello stesso collegio una percentuale di voti proporzionali $(14.9 \%)$ superiore a quella ottenuta da Michelini. Questa differenza però può essere considerata solo un indizio. 
È noto che i sistemi maggioritari a un turno sono associati ad un maggior tasso di astensionismo in quanto una quota di elettori non vota piuttosto che votare un candidato preferito che non è competitivo (e quindi sicuramente perdente) o un candidato competitivo, ma poco gradito. A questi elettori il voto di approvazione offre la possibilità di votare entrambi questi candidati. Questo rappresenta un incentivo importante a votare perché risolve almeno in parte il dilemma tra identità (voto per il partito di appartenenza) e razionalità (voto per il partito meno gradito, ma più competitivo) che caratterizza il comportamento dei sostenitori di partiti marginali e che li spinge verso l'astensione.

In conclusione, il voto di approvazione è un sistema più flessibile che offre agli elettori un maggior numero di opzioni rispetto al maggioritario semplice: votare per un unico candidato preferito a tutti gli altri oppure votare per più candidati considerati accettabili quando non si voglia o non si possa esprimere un'unica preferenza. Questa maggiore flessibilità del sistema produce un esito che riflette meglio le preferenze complessive del corpo elettorale perché, così come avviene con il metodo Condorcet, le preferenze che contano non sono solo le prime. Il risultato è una maggiore legittimità dell'esito del processo elettorale.

Questi sono gli argomenti più forti avanzati dai sostenitori del voto di approvazione. Sono argomenti convincenti (a parte le considerazioni che faremo tra breve), soprattutto se - come abbiamo fatto in queste pagine - il termine di confronto è il sistema maggioritario a un turno. Brams e Fishburn (1983) sostengono con forza la tesi della superiorità del voto di approvazione anche rispetto al maggioritario a due turni con ballottaggio. $\mathrm{Ma}$ il punto sostanziale è un altro. È vero che la formula ha delle potenzialità interessanti, ma è anche vero che ha dei limiti che occorre evidenziare per sfruttare appieno le prime e individuare meglio i possibili ambiti di applicazione.

Tra i limiti, il più importante è rappresentato senza dubbio dalla «condizione delle preferenze dicotomiche». Se e soltanto se le preferenze degli elettori soddisfano questa condizione si può affermare che il voto di approvazione è «il solo sistema elettorale non basato sull'ordinamento delle preferenze, che assicura l'elezione del candidato Condorcet, ammesso che tale candidato esista» (Brams e Fishburn 1983, 36) ${ }^{13}$. 
Che cosa significa «preferenze dicotomiche»? Perché sia soddisfatta questa condizione occorre che gli elettori dividano i candidati solo in due insiemi: l'insieme dei candidati preferiti (A) e quello dei candidati sgraditi (B). Inoltre, mentre tutti i candidati dell'insieme A sono preferiti a tutti i candidati dell'insieme $B$, nessun candidato dell'insieme $A$ è preferito agli altri candidati dello stesso insieme. La stessa relazione di indifferen$z a$ vale per i candidati dell'insieme $B$.

In questo caso ogni elettore ha un'unica strategia ammissibile, qualunque siano il numero dei candidati e le preferenze degli altri elettori ${ }^{14}$. Questa strategia è quella di votare tutti $\mathrm{i}$ candidati compresi nell'insieme A (quello dei candidati accettabili). Il risultato sistemico è l'elezione del candidato più approvato, cioè più accettabile, dalla maggioranza degli elettori che, data questa condizione, è il candidato Condorcet. Nessun'altra strategia è ammissibile e quindi nessun'altra strategia garantirebbe all'elettore un esito migliore, cioè l'elezione di un candidato a lui più gradito. Questa proprietà del sistema esclude qualunque incentivo ad un comportamento strategico. In altre parole, se nessuno dei candidati approvati da un elettore vince, nessuno di questi candidati avrebbe comunque potuto vincere se i loro sostenitori si fossero comportati strategicamente invece che sinceramente.

In questo caso, il voto di approvazione non è soltanto un «sistema elettorale sincero», ma anche un sistema elettorale «non manipolabile» ${ }^{15}$. Questa seconda proprietà è ancora più importante della prima (la sincerità). Infatti, un sistema elettorale è sincero se tutte le strategie di voto ammissibili sono sincere $^{16}$, qualunque siano le preferenze degli elettori. Ma un siste-

sostengono che il voto di approvazione, pur non eliminandolo, individua il candidato accettabile alla maggior parte degli elettori.

${ }^{14}$ Una strategia è ammissibile se e soltanto se è permessa dal sistema elettorale e se non esiste nessuna altra strategia permessa che la domina. Dal punto di vista di un elettore di riferimento, una strategia è dominante se e soltanto se porta a degli esiti buoni almeno quanto quelli di qualunque altra strategia per tutte le possibili configurazioni di preferenze elettorali, e ad un esito migliore per almeno una configurazione.

15 Traduco così il termine inglese «strategy-proof». Un sistema elettorale è non manipolabile se e soltanto se una sola strategia è ammissibile per ciascun elettore (nel quale caso questa strategia deve anche essere sincera).

${ }_{16}$ In generale, intendiamo per voto sincero, un voto che riflette le vere preferenze degli elettori. In termini più precisi, una strategia di voto di un qualunque elettore è sincera se e soltanto se comprende il votare per tutti i candidati preferiti al candidato meno preferito incluso nella strategia ammissibile dell'elettore in questione. In altre pa- 
ma sincero può offrire più di una strategia ammissibile a seconda del numero dei candidati in gara e della struttura di preferenza degli elettori. Quando questo accade, all'elettore resta il problema di scegliere una strategia di voto fra tutte quelle ammissibili. E questa scelta è influenzata in modo determinante dall'informazione che l'elettore ha sulle preferenze degli altri elettori nel collegio, cioè sul come votano gli altri.

In assenza di informazioni, qualunque strategia ammissibile è «buona». Ma il punto è un altro: quando il sistema elettorale è «manipolabile», esiste un incentivo importante per i candidati (e i partiti) a fornire informazioni sulla loro forza presunta ed esiste un incentivo altrettanto forte per gli elettori a cercare e usare queste informazioni per scegliere la migliore tra le strategie ammissibili, cioè per ottimizzare l'esito della competizione elettorale nel loro interesse. Il sistema allora è manipolabile.

Solo la proprietà della non manipolabilità (e non quella della sincerità) elimina il problema dell'informazione (sul come votano gli altri, cioè sulla consistenza delle altre candidature) e con ciò elimina qualunque incentivo a comportamenti strategici. Se il sistema è non manipolabile, tutti gli elettori hanno una sola strategia ammissibile: quella di votare tutti i candidati preferiti (insieme A) e quindi non hanno motivo né di cercare informazioni né di votare strategicamente.

E chiaro quindi che questa è una proprietà altamente desiderabile. Però è una proprietà che il voto di approvazione possiede se e soltanto se le preferenze sono dicotomiche. A questa condizione (e solo a questa condizione) è il migliore dei sistemi possibili: il candidato eletto è il candidato Condorcet; gli elettori non devono fare calcoli strategici complicati, ma possono votare $\mathrm{i}$ candidati che veramente preferiscono senza sacrificarne nessuno; i candidati, i partiti, la stampa, i sondaggi non possono influenzare il comportamento degli elettori e quindi l'esito delle elezioni; né questo esito può essere influenzato dalla presentazione di candidature di disturbo ${ }^{17}$.

$\mathrm{Ma}$ in tutti gli altri casi? Cosa succede quando le preferenze non sono dicotomiche? Nella realtà questa è una condizione

role, si deve parlare di strategia di voto sincera quando l'elettore che vota per il candidato A vota anche per tutti $i$ candidati preferiti ad A. Da questa definizione discende quella di sistema elettorale sincero. Un sistema elettorale è sincero se e soltanto se tutte le strategie ammissibili per gli elettori sono sincere, qualunque siano le loro preferenze.

${ }_{17}$ Questo perché il voto di approvazione è insensibile al numero di candidati. 
fortemente restrittiva. Infatti, passando dal piano analitico a quello empirico, può anche essere vero che in determinate situazioni gli elettori dividano $\mathrm{i}$ candidati in due sole classi e che siano indifferenti rispetto ai candidati dell'insieme $\mathrm{A}$ (così come dell'insieme B), ma in molte situazioni reali è probabile che essi preferiscano in assoluto la vittoria di un candidato e che non siano indifferenti rispetto agli altri, cioè a dire che tra questi altri ci siano candidati accettabili (ma non quanto il candidato maggiormente preferito) e assolutamente non accettabili. Una struttura di preferenze di questo tipo non è dicotomica, ma tricotomica. E allora i meriti del voto di approvazione, pur non venendo completamente meno, si attenuano fortemente ${ }^{18}$.

Prendiamo come esempio il solito collegio di Roma-Centro. Perché il voto di approvazione funzionasse in maniera ottimale occorrerebbe ipotizzare una struttura di preferenze come questa (dove le parentesi racchiudono i candidati dell'insieme A e le percentuali di voto sono quelle della tabella 1 ):

$\begin{array}{ccc}40,1 \% & 12,8 \% & 46,3 \% \\ \text { (Spaventa, Michelini) } & \text { Michelini } & \text { Berlusconi } \\ \text { Berlusconi } & \text { (Spaventa, Berlusconi) } & \text { (Michelini, Spaventa) }\end{array}$

In questo caso, con il voto di approvazione sarebbe stato eletto Michelini, invece di Berlusconi. Ma è molto probabile che questa struttura di preferenze sia irrealistica. Quanti di quel $40 \%$ di elettori che ha votato Spaventa consideravano Michelini come un candidato ugualmente accettabile? Sicuramente non tutti. E allora basta questa deviazione dal modello per entrare in un altro ordine di ragionamento centrato sulla domanda: sarebbe stato veramente conveniente per gli elettori di Spaventa esprimere comunque un secondo voto a favore di Michelini per evitare l'elezione di Berlusconi? Ex post, si potrebbe rispondere affermativamente. Ma ex ante la situazione decisionale si presenta in maniera del tutto diversa. L'assenza di informazioni sulla consistenza delle varie candidature modifica la struttura di

18 Brams e Fishburn $(1983,30)$ sostengono che, se le preferenze sono tricotomiche [esempio: $\mathrm{A}(\mathrm{BC}) \mathrm{D}$ ], allora il voto di approvazione è ancora un sistema sincero e che in ogni caso esso è il più sincero tra tutti i sistemi non basati su ordinamento delle preferenze. Su questo punto, però, vedi Niemi (1984) il quale ha dimostrato efficacemente (e criticamente) quali sono le proprietà che caratterizzano il voto di approvazione se le preferenze non sono dicotomiche. 
opportunità degli elettori di Spaventa. Più precisamente, il fatto che le strategie di voto ammissibili siano più di una offre a questi elettori un incentivo ad informarsi sul come votano gli altri e offre ai candidati un incentivo analogo a fornire dati (non si sa quanto accurati) sulla loro forza elettorale. Ed è proprio questo tipo di informazioni che può orientare la decisione degli elettori di Spaventa se votare solo Spaventa o anche Michelini. Soltanto, nell'ipotesi dell'informazione perfetta, il sistema elettorale non sarebbe manipolabile nel caso in cui le preferenze degli elettori di Spaventa invece di essere dicotomiche, fossero tricotomiche (cioè, Spaventa preferito a Michelini e Michelini preferito a Berlusconi, invece che Spaventa $e$ Michelini ugualmente preferiti a Berlusconi).

In altri termini, se gli elettori di Spaventa avessero ipotizzato che il loro candidato non era competitivo, allora certamente sarebbe convenuto loro votare anche Michelini per contribuire alla vittoria di un candidato meno gradito del loro, ma più gradito di Berlusconi. Se, invece, tali elettori fossero stati convinti della competitività della candidatura di Spaventa, votando Michelini avrebbero sì contribuito alla sconfitta di Berlusconi, ma anche alla sconfitta dello stesso Spaventa a favore di Michelini. L'esempio dimostra che, se non vale la condizione delle preferenze dicotomiche, allora diventa cruciale la posizione relativa dei candidati nella gara (variabile-competitività) e la percezione di questa posizione da parte degli elettori (variabile-informazione).

Questo si può dire: con il voto di approvazione, i sostenitori di candidati estremi o comunque poco competitivi hanno sempre un incentivo in un modello di scelta razionale a votare anche un altro candidato meno estremo o meno marginale. In questo modo si produce quell'effetto centripeto che è tipico dei sistemi elettorali che utilizzano, insieme alle prime, anche le seconde preferenze degli elettori. Un po' come succede al secondo turno in un sistema maggioritario a due turni. In altre parole, il voto di approvazione dato al candidato preferito in assoluto equivale al voto dato nella prima tornata del sistema maggioritario a due turni. L'elettore vota il partito di appartenenza e questo voto serve a contabilizzare il sostegno per i partiti marginali e/o estremi. Il secondo voto dato al candidato «approvato», ma non preferito in assoluto equivale al voto dato al secondo turno al candidato meno sgradito. In entrambi i casi si produce un effetto centripeto con la vittoria dei candidati più mo- 
derati, più centrali, capaci di raccogliere un sostegno più ampio dei candidati collocati nelle posizioni estreme dello schieramento politico e/o dei candidati portatori di interessi settoriali.

Esiste però una differenza importante tra voto di approvazione e maggioritario a due turni (con o senza ballottaggio): con il primo sistema gli elettori votano «al buio», vale a dire senza avere l'informazione fornita loro dai risultati del primo turno relativamente alla consistenza delle varie candidature. Se le preferenze sono dicotomiche, questa differenza non conta e allora si può dire che il voto di approvazione è un doppio turno ridotto ad un turno solo; di più, si può affermare con maggior precisione che il voto di approvazione diventa un sistema maggioritario ad un turno con ballottaggio, dove il ballottaggio anziché essere tra due candidati è tra due insiemi di candidati.

Se invece le preferenze non sono dicotomiche (e questa è l'ipotesi più probabile nella maggioranza delle situazioni reali), allora il votare in due turni anziché in uno diventa molto importante perché risolve in parte il problema della manipolabilità del sistema elettorale fornendo agli elettori delle informazioni in base alle quali decidere quale tra le strategie ammissibili adottare. Tant'è che possiamo chiederci se il sistema «ideale» non sarebbe un sistema a due turni che prevedesse la regola della maggioranza assoluta al primo turno e il voto di approvazione al secondo turno.

Per esempio, nel caso del collegio di Roma-Centro, tutti gli elettori avrebbero votato la loro prima preferenza al primo turno senza dover ricorrere a rassemblements dicotomici o calcoli strategici. Se nessun candidato avesse ottenuto la maggioranza assoluta dei voti, si sarebbe passato al secondo turno con voto di approvazione. Qui ciascun elettore avrebbe potuto decidere, alla luce dei risultati del primo turno, se votare uno o due candidati. Con questa procedura si attenuerebbe il vincolo delle preferenze dicotomiche; diventa infatti più probabile che si produca un effetto dicotomico per cui il secondo turno potrebbe forse diventare quel ballottaggio tra due insiemi di candidati, $i$ buoni e i cattivi, di cui abbiamo parlato sopra.

\section{Conclusioni}

Il voto di approvazione non è il sistema ideale. Forse il sistema ideale non esiste. Esistono invece dei criteri per orientarsi 
nella scelta dei sistemi elettorali. Uno di questi criteri è quello di Condorcet. Altri due che abbiamo trattato in questa sede sono quelli della sincerità e della non manipolabilità. Sono criteri importanti, anche se siamo pronti ad ammettere che non siano gli unici per valutare meriti e demeriti di sistemi elettorali del mondo reale (oltre che per ragionare astrattamente sulle procedure di voto). Siamo però convinti che questi criteri (e in particolare il criterio di Condorcet) siano criteri forti dai quali non si può prescindere senza giustificazioni. Un sistema elettorale che, a parità di condizioni, soddisfi questi criteri meglio di un altro è preferibile.

Il voto di approvazione soddisfa bene questi criteri se le preferenze di tutti gli elettori sono dicotomiche. Ma quando è applicabile questa condizione? In quali casi è più probabile che gli elettori tendano a dividere $i$ candidati in due classi soltanto? Questa è la questione importante da un punto di vista empirico. Rispondere a questa domanda vuol dire individuare i campi di applicazione di questa nuova formula. Riker $(1983,113)$ sostiene che siano le elezioni primarie il miglior terreno di sperimentazione. Gli stessi Brams e Fishburn aderiscono a questa ipotesi (1983; 1984), pur senza considerarla l'unica. Probabilmente ci sono altri ambiti, per esempio i congressi di partito, nei quali sarebbe interessante applicare il voto di approvazione.

Un'altra possibilità potrebbe essere quella - suggerita timidamente alla fine del paragrafo precedente - di un sistema a due turni con regole differenziate (maggioritario al primo turno e con voto di approvazione al secondo). Chi scrive, però, è personalmente convinto che nella maggior parte delle situazioni pratiche a livello di elezioni politiche generali - e certamente in questo paese in questa fase storica - il doppio turno «classico» con o senza ballottaggio sia superiore, in base ai criteri e per i motivi utilizzati qui, sia al turno singolo che al voto di approvazione. Quest'ultimo resta un contributo interessante alla teoria dei sistemi elettorali oltre che un'idea in cerca di applicazione.

\section{Riferimenti bibliografici}

Arrington S.T. e S. Brenner (1984), Another Look at Approval Voting, in «Polity», XVII, pp. 118-134.

Arrow K.J. (1963), Social Choice and Individual Values, New Haven, Yale University Press; trad. it. Scelte sociali e valori individuali, Milano, Etas Libri, 1977. 
Black D. (1958), The Theory of Committees and Elections, Cambridge, Cambridge University Press.

Borda, J.C. de (1781), Memoire sur les elections au scrutin, Histoire de l'Academie Royale des Sciences, Parigi.

Brams S.J. e P.C. Fishburn (1983), Approval Voting, Boston, Birkhauser.

- (1984), A Careful Look at «Another Look at Approval Voting», in «Polity», XVII, pp. 135-143.

Condorcet, Marquis de (1785), Essai sur l'application de l'analyse a la probabilité des decisions rendues à la pluralité des voix, Parigi.

Cox W.G. (1985), Electoral Equilibrium under Approval Voting, in «American Journal of Political Science», XXIX, pp. 112-118.

D'Alimonte R. e A. Chiaramonte (1993), Il nuovo sistema elettorale italiano: quali opportunità?, in «Rivista Italiana di Scienza Politica», XXIII, pp. 513-548.

Niemi R.G. (1984), The Problem of Strategic Behavior under Approval Voting, in «American Political Science Review», LXXVIII, pp. 952-958.

Nurmi H. (1983), Voting Procedures: A Summary Analysis, in «British Journal of Political Science», XII, pp. 181-208.

Riker W.H. (1983), Liberalism Against Populism: A Confrontation Between the Theory of Democracy and the Theory of Social Choice, San Francisco, W.H. Freeman.

Wright J.F.H. (1986), Australian Experience with Majority-Preferential and Quota-Preferential Systems, in B. Grofman e A. Lijphart (a cura di), Electoral Laws and Their Political Consequences, New York, Agathon Press, pp. 124-138. 